modality with corresponding survival are shown in Table 1. $243(10.5 \%)$ patients received surgery and chemotherapy, 230 $(10 \%)$ patients received radical radiotherapy and chemotherapy, $618(26.8 \%)$ palliative radiotherapy or palliative chemotherapy and $802(34.8 \%)$ received best supportive care. The proportion of patients alive was $74.4 \%$ in patients receiving surgery; $63.2 \%$ for patients receiving radical radiotherapy, $41.8 \%$ for palliative chemotherapy/radiotherapy and $23.1 \%$ for supportive care.

Conclusions The commonest curative intent treatments are bimodality treatment (chemotherapy combined with either surgery or radical radiotherapy), however only one fifth of patients received this. The majority of patient still receive palliative treatment only. Survival is higher in patients who receive surgery as part of their treatment however we are unable to exclude selection bias as the reason for this. Further risk adjustment analysis will be performed to assess this.

\section{S99 LUNG CANCER SURGICAL OUTCOMES IN ENGLAND (2015)}

${ }^{1} \mathrm{P}$ Beckett, ${ }^{1} \mathrm{R}$ Dickinson, ${ }^{2} \mathrm{R}$ Hubbard, ${ }^{2} \mathrm{~A}$ Khakwani, ${ }^{3} \mathrm{D}$ West. ${ }^{1}$ Royal College of Physicians, London, UK; ${ }^{2}$ University of Nottingham, Nottingham, UK; ${ }^{3}$ Society of Cardiothoracic Surgeons, London, UK

\subsection{6/thoraxjnl-2017-210983.105}

Introduction The National Lung Cancer Audit has collected data for over 10 years demonstrating gradually rising resection rates in the UK. The Clinical Outcomes Programme (COP) uses national audit data to publish quality measures at the level of individual consultants. The lung cancer COP focusses on activity at individual surgeon level, and on survival at unit level. Previous rounds of this audit demonstrated overall 30

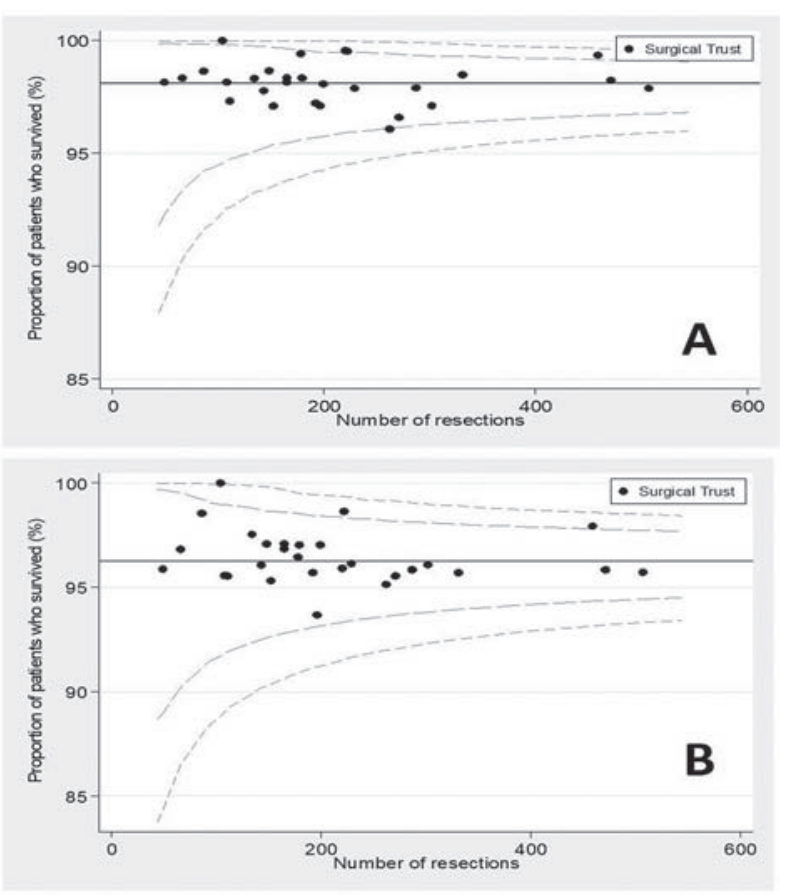

Abstract S99 Figure 1 Funnel plots of adjusted survival for 30 days (A) and 90 days (B) survival after surgery for lung cancer in English trusts. and 90 day survival of $97.8 \%$ and $95.5 \%$ (2013) and $97.9 \%$ and $96.2 \%$ (2014).

Methods Data submitted to the NLCA for patients having curative-intent surgery who underwent surgery in 2015 was sent to the clinical lead at each surgical unit for validation and addition of responsible surgeon GMC number, with the option to add surgical cases if they were not included in the supplied dataset. Date of death was derived by a link to the Office of National Statistics. Units reporting unadjusted survival proportions more than three standard errors outside the national mean ("alarm" level) at 30 or 90 days were identified as statistical outliers.

Results All of the 28 surgical units in England participated in the audit, submitting a total of 5843 cases, of which $93 \%$ were NSCLC, 2\% SCLC and 6\% carcinoid. Median annual unit activity was 186 resections (IQR 141-264, range 49507), an increase over the median 156 in the previous year. Median annual activity for individual surgeons was 44 (IQR 18-67, range 1-171). Median LOS 8.1 days (median 6, IQR 4-9). Overall 30 day survival was $98.2 \%$ and 90 day survival was $96.3 \%$. There were no units with statistical outliers at the alarm level at 30 days and 90 days (see figure 1). Overall 1 year survival $87.9 \%$.

Conclusion Volume of activity varies widely by unit and individual surgeon. Survival after lung cancer surgery is very high, is improving, and is not statistically significantly different across the surgical units in England. New measures are needed to properly reflect features that are important to patients.

\section{S100 UTILITY OF ENDOBRONCHIAL ULTRASOUND-GUIDED TRANSBRONCHIAL NEEDLE ASPIRATION FOR PD-L1 TESTING IN PATIENTS WITH NSCLC}

${ }^{1} \mathrm{~F}$ Perrotta, ${ }^{2} \mathrm{~B}$ Adizie, ${ }^{3} \mathrm{U}$ Maqsood, ${ }^{4} \mathrm{M}$ Elshafi, ${ }^{5} \mathrm{~S}$ Jafri, ${ }^{2}$ I Woolhouse, ${ }^{3} \mathrm{M}$ Munawar, ${ }^{4} \mathrm{M}$ Evison, ${ }^{4} \mathrm{R}$ Booton, ${ }^{5} \mathrm{DR}$ Baldwin, ${ }^{6} \mathrm{SM}$ Janes, ${ }^{1} \mathrm{~A}$ Bianco, ${ }^{6} \mathrm{~N}$ Navani. ${ }^{1}$ University of Campania "L. Vanvitelli", Naples, Italy; ${ }^{2}$ University Hospitals Birmingham, Birmingham, UK; ${ }^{3}$ Lancashire Teaching Hospitals, Preston, UK; ${ }^{4}$ University Hospital of South Manchester, Manchester, UK; ${ }^{5}$ Nottingham University Hospitals, Nottingham, UK; ${ }^{6}$ University College of London, London, UK

\subsection{6/thoraxjnl-2017-210983.106}

Rationale Recent data have demonstrated the superiority of Pembroluzimab over chemotherapy for patients with advanced NSCLC and high ( $\geq 50 \%$ expression) of PD-L1.1 This has resulted in NICE approving Pembroluzimab as a first line treatment option for patients with advanced NSCLC in June 2017. The original trial however excluded patients with PDL1 testing on EBUS samples. We therefore conducted a large, multicentre study to clarify whether specimens obtained by EBUS-TBNA were suitable for testing PD-L1 in patients with NSCLC.

Methods NSCLC samples acquired by EBUS-TBNA (29.4\%), percutaneous biopsy (31.2\%), endobronchial biopsy (13.8\%), surgical $(21.4 \%)$ or other techniques $(4.1 \%)$ were recorded from 435 consecutive patients with known or suspected lung cancer across 5 centres in England between January 2015 and December 2016.

Results PD-L1 assessment (using the $22 \mathrm{C} 3$ assay in all cases) was possible in $92.2 \%$ of patients undergoing EBUS and there was no difference in success of PD-L1 testing according to modality of tissue acquisition $(p=0.18)$. The frequency of complications from EBUS-TBNA was similar to endobronchial or percutaneous techniques but lower than surgical procedures 